Signal quality of endovascular electroencephalography

This content has been downloaded from IOPscience. Please scroll down to see the full text. 2016 J. Neural Eng. 13016016

(http://iopscience.iop.org/1741-2552/13/1/016016)

View the table of contents for this issue, or go to the journal homepage for more

Download details:

IP Address: 131.215.70.231

This content was downloaded on 12/01/2016 at 18:25

Please note that terms and conditions apply. 


\title{
Signal quality of endovascular electroencephalography
}

\author{
Bryan D He ${ }^{1,2,3}$, Mosalam Ebrahimi ${ }^{1,3}$, Leon Palafox ${ }^{1}$ and \\ Lakshminarayan Srinivasan ${ }^{1}$ \\ ${ }^{1}$ Neural Signal Processing Laboratory, Department of Radiology, Stanford University, Stanford, CA \\ 94305, USA \\ ${ }^{2}$ Department of Computer Science, California Institute of Technology, Pasadena, CA 91125, USA \\ E-mail: 1s2@nsplab.org
}

Received 11 March 2015, revised 2 September 2015

Accepted for publication 18 September 2015

Published 6 January 2016

\begin{abstract}
Objective, Approach. A growing number of prototypes for diagnosing and treating neurological and psychiatric diseases are predicated on access to high-quality brain signals, which typically requires surgically opening the skull. Where endovascular navigation previously transformed the treatment of cerebral vascular malformations, we now show that it can provide access to brain signals with substantially higher signal quality than scalp recordings. Main results. While endovascular signals were known to be larger in amplitude than scalp signals, our analysis in rabbits borrows a standard technique from communication theory to show endovascular signals also have up to $100 \times$ better signal-to-noise ratio. Significance. With a viable minimally-invasive path to high-quality brain signals, patients with brain diseases could one day receive potent electroceuticals through the bloodstream, in the course of a brief outpatient procedure.
\end{abstract}

Keywords: endovascular neural interface, signal quality

(Some figures may appear in colour only in the online journal)

\section{Introduction}

The detailed spatial and temporal dynamics of electric potentials recorded from intracranial electrodes predict motor intent, detect seizure onset, and reflect the dynamics of memory and decision making. To date, neuroscience has largely relied on skull perforation or skull opening to place electrodes that access neural signals with kilohertz-wide frequency content, millisecond temporal resolution, and submillimeter spatial resolution. The risks and drawbacks of neurosurgery to achieve intracranial electroencephalography (iEEG) limits the clinical application of sophisticated algorithms based on these electrodes to a subset of patients and experimental settings. From 2004 to 2009 in the United

${ }^{3}$ Equal contribution.

Content from this work may be used under the terms of the Creative Commons Attribution 3.0 licence. Any further distribution of this work must maintain attribution to the author(s) and the title of the work, journal citation and DOI.
States, a total of 101123 patients were hospitalized for epilepsy. Of these patients, 40\% (40 942) received scalp EEG monitoring and only 6\% (6422) underwent intracranial EEG monitoring, with a comparable percentage of patients going on to epilepsy surgery (Schiltz et al 2013). With the recent emergence of promising new surgical techniques for the management of epilepsy (Nowell et al 2014), access to definitive diagnosis through intracranial recording may represent a chokepoint in disseminating these compelling therapies.

Intracranial electrodes, including subdural and depth electrodes, are employed in surgical planning when ambiguity remains with non-invasive methods (EEG, MEG, MRI, SPECT, PET) (Shah and Mittal 2014). Applications include mapping of suspected medial temporal lobe epilepsy, stimulator implantation in subthalamic nucleus, and intraoperative functional mapping of language areas during tissue resection. Bypassing scalp, skull, and dura, iEEG preserves a wide range of frequency content (beyond $500 \mathrm{~Hz}$ ), where spontaneous scalp EEG degrades above $50 \mathrm{~Hz}$ (Schalk and Leuthardt 2011). Scalp EEG frequency content broadens where skull has been previously removed to relieve brain swelling 
(Voytek et al 2010). Source localization using subdural iEEG affords millimeter-scale spatial resolution, compared with centimeter-scale resolution using scalp EEG (Schalk and Leuthardt 2011).

Despite these advantages, iEEG presents known challenges for the patient, tangible medical risks, and technical limitations. Considerable emotional stress and post-operative headache are routinely encountered with neurosurgical intervention (Lozano et al 2009). Depth electrodes involve drilling burr holes into the skull, and subdural grid electrodes typically require wider craniotomy. Intracranial bleed, infection, and edema along electrode tracts are known risks, with rates between $2 \%$ and $20 \%$ depending on the definition of complication (Wellmer et al 2012, Arya et al 2013). For comparison, rates of complication with DBS electrodes are 3\% for hemorrhage (Ben-Haim et al 2009) and 1\% for infection, and as high as $10 \%$ if leads are temporarily externalized (Chan et al 2009). Large subdural grids ( $>67$ electrodes) are more prone to adverse events (Arya et al 2013). The rates for depth electrodes are lower in comparison with subdural strip and grid electrodes (Wellmer et al 2012). Repositioning grids or depth electrodes through revision surgery based on initial recordings is not practical. For select applications including mesial temporal sclerosis, foramen ovale electrodes provide intracranial recordings without craniotomy, but these recordings are restricted to the ambient cistern near the skull base (Sheth et al 2014), with risks including damage to the trigeminal nerve, infection, and bleeding.

Modern techniques allow physicians to safely traverse cerebral vessels to achieve a full range of anatomical positions from the skull base to the cortical surface and throughout the brain. With roughly 800 neurointerventionalists across 400 hospitals in the United States (Fiorella et al 2012), sub-millimeter guidewires and catheters are now routinely navigated through arterial and venous cerebral vasculature. Traditionally, advances using these techniques have focused almost exclusively on treating vascular pathology, including aneurysms, malformations, fistulas, and stroke. For many patients with these conditions, endovascular procedures have spared them open-skull surgery and prolonged hospital stay. The progressive refinement of these techniques has reduced the risk of intracranial complications (primarily related to stroke) to $3 \%$ from microcatheter intervention and $0.07 \%$ from diagnostic cerebral angiography (Cloft et al 1999).

Although endovascular EEG is not presently in clinical use, the possibility of recording iEEG through vessels that supply the brain has been the subject of intermittent exploration over the past four decades (Driller et al 1968, Penn et al 1973, Zeitlhofer et al 1990, Nakase et al 1995, Stoeter et al 1995, Boniface and Antoun 1997, Mikuni et al 1997, Ishida et al 1998, Thomke et al 1998, GarcíaAsensio et al 1999, Kunieda et al 2000, Enomoto et al 2002, Llinas et al 2005, Ruddy 2006, Watanabe et al 2009, Bower et al 2013, Henz et al 2014), including related patent applications (Litt and Echauz 2007, Llinas 2007, Asirvatham et al 2012). Composed variously of platinum and nitinol alloys, the guidewires that facilitate endovascular access are conductive, atraumatic, biologically inert and torquable.
When passed into the cerebral vasculature of the human brain, these guidewires record evoked potentials with substantially larger magnitude than scalp potentials. Guidewires have been left within venous sinuses for prolonged recording in an epilepsy monitoring unit (Mikuni et al 1997). Recent animal models have reproduced these findings with platinum electrodes. In one study, platinum electrode strips were surgically placed in the superior sagittal sinus of sheep to record penicillin-induced ictal waveforms (Bower et al 2013). Similar unpublished work has been performed by others. In the aforementioned studies, signal amplitudes resembled those of subdural iEEG. In contrast, evoked potentials recorded from peripheral nerves are comparable in amplitude between endovascular and skin-surface recordings, where interposed skull is not present to impede dermal EEG (Llinas et al 2005).

Despite this literature on endovascular EEG, the fundamental question of signal quality remains unanswered. This is because signal amplitude alone does not determine the basic measures of signal quality that are important to iEEG applications, including signal-to-noise ratio (SNR), frequency content, and spatial sensitivity. With regards to signal variability (in terms of SNR), there are several practical reasons for concern. Mechanical pulsation artifact is known to degrade subdural electrodes that are located in proximity to cortical arteries, and this effect could be magnified within the vessel (Simon 2009). Cardiac field artifact in subdural electrodes could be more pronounced with guidewire electrodes that pass through the chest cavity en route to the brain (Kern et al 2013). Scalp EEG and transcranial iEEG leads are kept short to avoid electrical interference, but guidewires are nearly two orders of magnitude longer at 175 to $200 \mathrm{~cm}$. With regards to frequency content, the impedance spectra of guidewire metal alloys are uncharacterized, where $\mathrm{Ag} / \mathrm{AgCl}$ and platinum are proven electrode materials applied in scalp EEG and subdural iEEG respectively (Nelson et al 2008). With regards to spatial sensitivity, a systematic evaluation of stereotyped neural response for comparison across endovascular positions recorded within millimeters is unavailable to show advantage over scalp recordings. Finally, basic live and dead-animal controls are missing from the nascent literature on endovascular EEG to confirm that stimulus-driven responses are biological in origin rather than electronicsinduced artifact.

As a practical matter, the lack of small animal models presents a logistical barrier to the development of endovascular electrophysiology. Previous demonstrations were performed in man (Penn et al 1973, Zeitlhofer et al 1990, Nakase et al 1995, Stoeter et al 1995, Boniface and Antoun 1997, Mikuni et al 1997, Ishida et al 1998, Thomke et al 1998, García-Asensio et al 1999, Kunieda et al 2000, Enomoto et al 2002), baboon (Penn et al 1973), swine (Bower et al 2013), and sheep (Litt and Echauz 2007), subjects which are not widely supported across research facilities. The New Zealand White rabbit represents an economical small-animal alternative to these models with anthropomorphic cerebral vasculature (Scremin et al 1982, Caldwell et al 2011) and coagulation cascade (Mehra et al 2012). Structural (MuñozMoreno et al 2013, Müllhaupt et al 2015) and functional 
(Wyrwicz et al 2000) magnetic resonance imaging (MRI) of the rabbit brain have been performed. Three-plane (Shek et al 1986) and stereotactic (Richard and Urban 1972) anatomical atlases are available. Nevertheless, trans-femoral endovascular intracranial access with microcatheters is rarely attempted in rabbit (Culp et al 2007, Jahan et al 2008) because the cerebral vasculature is $2-4$ times smaller than human and rabbit cerebral arteries are easily prone to vasospasm (Culp et al 2007). Additionally, literature on the various rabbit evoked potentials (including stimulus parameters and neural mechanism) is limited (Arezzo et al 1988, Hori et al 1992, 1993), and endovascular recording of evoked potentials in rabbit is unproven.

In this paper, we develop the rabbit model and demonstrate the viability of endovascular EEG in terms of fundamental measures of signal quality, including SNR, frequency content, and spatial sensitivity. Endovascular guidewire electrode intracranial EEG recordings are achieved via a clinically-realistic trans-femoral approach. Various evoked potentials are employed to provide a repeatable test of signal quality, including visual evoked potential (VEP), steady state auditory evoked potential (SSAEP), and steady state visual evoked potential (SSVEP). Using simultaneously acquired electrocardiogram, endovascular contamination from cardiac potentials is demonstrated and removed. Signals are examined in the time and frequency domains. The experimental design incorporates both live and dead animal controls to confirm true stimulus-driven biological responses on the endovascular electrode rather than non-neural biological signal or stimulusinduced artifact. In vitro, electrochemical methods are used to characterize the frequency response of a standard guidewire in comparison with platinum, platinum black, and $\mathrm{Ag} / \mathrm{AgCl}$ electrodes. Taken together, these data demonstrate signal quality that justifies the ultimate goal of providing patients with an endovascular, minimally-invasive alternative to openskull electrophysiological brain mapping.

\section{Methods}

All procedures were approved by the University of California Chancellor's Animal Research Committee (protocol \# 2012079-02).

\section{Surgical preparation}

In each of two male New Zealand White rabbits, anesthesia was induced with ketamine $30 \mathrm{mg} \mathrm{kg}^{-1}$ and midazolam $1 \mathrm{mg} \mathrm{kg}^{-1} \mathrm{IM}$, and maintained using $1 \%-3 \%$ isoflurane gas via a $3.0 \mathrm{~mm}$ endotracheal tube. A 24 gauge indwelling catheter was placed in the marginal ear vein for fluid administration at $3-10 \mathrm{~mL} \mathrm{~kg}^{-1} \mathrm{~h}^{-1}$ during the procedure. A 5 French urinary catheter was also placed for subject comfort. Femoral access was achieved by first isolating the right femoral artery through an incision at the right femoral groove followed by blunt dissection. The exposed femoral artery was dilated by applying $2 \%$ lidocaine $(0.2-0.5 \mathrm{~mL})$ topically. A 4 French $(\mathrm{Fr})$ sheath was placed via serial dilation and Seldinger technique. This sheath was sutured to the isolated vessel segment using 2-0 silk. Heparinized saline (2 units Heparin/mL of saline) was introduced into the sidearm of the sheath at 1 drip per second to minimize risk of thrombus formation.

\section{Trans-femoral endovascular intracranial access and imaging}

Endovascular navigation was performed using single plane fluoroscopy (Siemens Artis zeego). A 4 Fr Terumo angled glidecatheter was advanced to the aortic arch over a $0.018^{\prime \prime}$ hydrophilic coated angled glidewire. From this position, digital subtraction angiography and roadmap were performed to define the arch anatomy, which was variable across subjects. The larger of the two vertebral arteries was selected and the glidecatheter was advanced to the osteum of the vertebral artery. Using a hemostatic valve with sidearm attached to the hub of the glidecatheter, an Echelon 10 microcatheter $\left(45^{\circ}\right.$ angled, 1.7 Fr tip) was advanced over an $0.010^{\prime \prime} \mathrm{X}$-Pedion-10 microwire through the glidecatheter and into the vertebral artery. A roadmap was performed via the microcatheter to define the cerebral vascular anatomy, including the intracranial basilar artery and circle of Willis (figure 1(A)). The microcatheter was then advanced delicately through the sharp turn of the vertebral artery over the $\mathrm{C} 1$ arch and positioned at the basilar tip with the guidewire just beyond the catheter tip (figure 1(B)). When vasospasm temporarily precluded traversal of the vertebral artery, efforts to navigate the microcatheter were suspended for approximately $10 \mathrm{~min}$ after which angiography was performed to demonstrate resolution of vasospasm and the procedure was resumed.

In the initial basilar tip position and subsequent recording positions at the mid-basilar artery and vertebrobasilar junction, cone-beam computed tomography (DynaCT) was performed using the fluoroscopy machine, to co-register guidewire electrode position with bony anatomy (figure 1(C)). Following sacrifice with Euthasol (pentobarbital sodium and phenytoin sodium), this bony anatomy was related to underlying brain parenchyma through post-mortem high-resolution T2-weighted MRI (figure 1(D)). Three-dimensional reconstruction from the CT data provides a global view of the endovascular approach (figure 1(E)).

\section{Evoked potentials}

Evoked potentials were recorded under general anesthesia. Each of three types of evoked potentials were examined in the rabbit: VEP (Arezzo et al 1988), SSAEP (Hori et al 1993), and SSVEP (Hori et al 1992, Herrmann 2001).

Recording system and electrode positioning. Evoked potentials were recorded at $9600 \mathrm{~Hz}$ using the g.Tech g.HIAmp data acquisition system with passive connectors. In addition to low-pass anti-aliasing filtering, high-pass filtering was applied to all signals, both cardiac and neural, with $10 \mathrm{~Hz}$ passband frequency, $2 \mathrm{~Hz}$ stopband frequency, $90 \mathrm{~dB}$ passband ripple, and $1 \mathrm{~dB}$ stopband attenuation. Scalp electrodes (Ag/AgCl pre-gelled, stick-on, $3 \mathrm{ft}$, Rochester Electromedical) were positioned posterior to the nose (reference), 


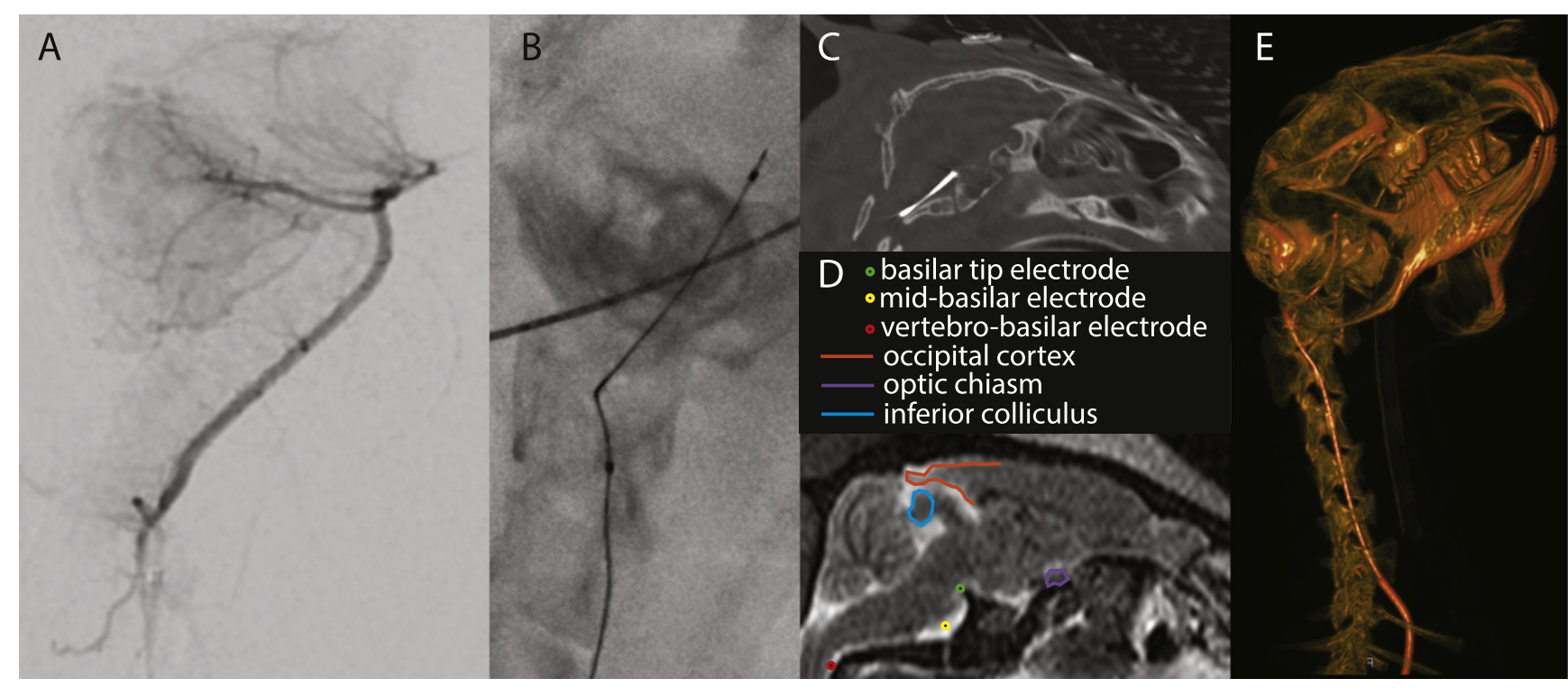

Figure 1. Anatomic localization and endovascular approach. Digital subtraction angiogram from microcatheter at distal vertebral artery demonstrates a lateral projection of the posterior circulation in arterial and early parenchymal phases, outlining the cerebellum and occipital cortex. The basilar artery, posterior cerebral arteries, posterior communicating arteries, and thalamic perforators opacify. (B) Lateral oblique spot view demonstrates the endovascular guidewire electrode projecting just beyond the tip of the microcatheter. (C) Sagittal reformat noncontrast cone-beam computed tomography (CT) performed from the uoroscopy table confirms positioning at the basilar tip relative to the postero-superior border of the clivus. This bony landmark permits co-registration of the three endovascular record-ing positions with postmortem T2-weighted MRI which demonstrates the locations of brain regions described in the generation of VEP, SSAEP, and SSVEP. (E) Three-dimensional re-constructed CT of the neck and head prior to basilar tip recording demonstrates the complete endovascular approach to the posterior circulation, including guide catheter, microcatheter, and guidewire electrode.

overlying the mid-head, and posterior to the bilateral eyes. Endovascular guide wire recording was performed using an alligator clip connected to the proximal-most segment of the guidewire, converting to a touch-proof connector input for the g.HIAmp head box. In case of poor electrical contact indicated by lack of cardiac activity or evoked potentials, various measures were performed including abrasion of the guidewire with the alligator clip, cleaning the guidewire with isopropyl alcohol wipes, and bending of the wire to fit tightly against the teeth of the clip. Care was taken to avoid excessive movement of the guidewire when attaching the alligator clip. Furthermore, both cross-sectional and planar fluoroscopic imaging were always obtained following attachment of the alligator clip to confirm positioning.

Resting state recordings. Repeated resting state recordings without stimulus were performed before all steady state evoked potential stimulus conditions. Resting state power spectral densities (figure 5) progressively diminished with the endovascular electrode, and remained stable for the scalp electrodes. This trend was recapitulated across both subjects. Tracking this non-stationarity was essential to computing valid SNR estimates for SSAEP and SSVEP conditions. The underlying cause for endovascular non-stationarity could be multifactorial, including causes related to the underlying biology or the chemistry of the electrode-electrolyte interface.

Live and dead controls. For each type of evoked potential, two separate controls were run. In live controls, the relevant stimulus was obstructed, and the stimulus was run. In the case of VEP and SSVEP, an opaque cardboard barrier was inserted between the LED and the right eye. In the case of SSAEP, the earbud was removed from the ear canal, tightly wrapped in cloth to attenuate the auditory stimulus, and placed along the right neck. The goal of the live control was to exclude electrical interference as an explanation for the observed trends. In the dead controls, unobstructed light or auditory stimulation was performed after sacrifice. The goal of the dead control was to additionally exclude non-brain biological interference as an explanation for the observed trends.

VEP. The VEP was elicited by placing a white LED at approximately $2 \mathrm{~cm}$ from the subject's right eye. The LED alternated between on and off states, each lasting $891 \mathrm{~ms}$. A digital input line on the data acquisition system recorded the LED state concurrently with neural recordings. After cardiac artifact removal (see below), single-trial evoked potentials were aligned to rising and falling edges of the LED, corresponding to On and Off responses. Trial-averaged response and bootstrapped confidence intervals for this average response were calculated. Between 153 and 208 single VEP trials were recorded for each trial-averaged response in figures 2 and 3. In figure 2, each trialaveraged response consists of between 205 and 208 single VEP trials for Subject 1 and 153 single VEP trials for Subject 2. In figure 3 , each trial-averaged response consists of between 191 and 208 single VEP trials for Subject 1.

SSVEP. The SSVEP was elicited by the same white LED right eye positioning as in the VEP, but now flashed at $40 \mathrm{~Hz}$ 

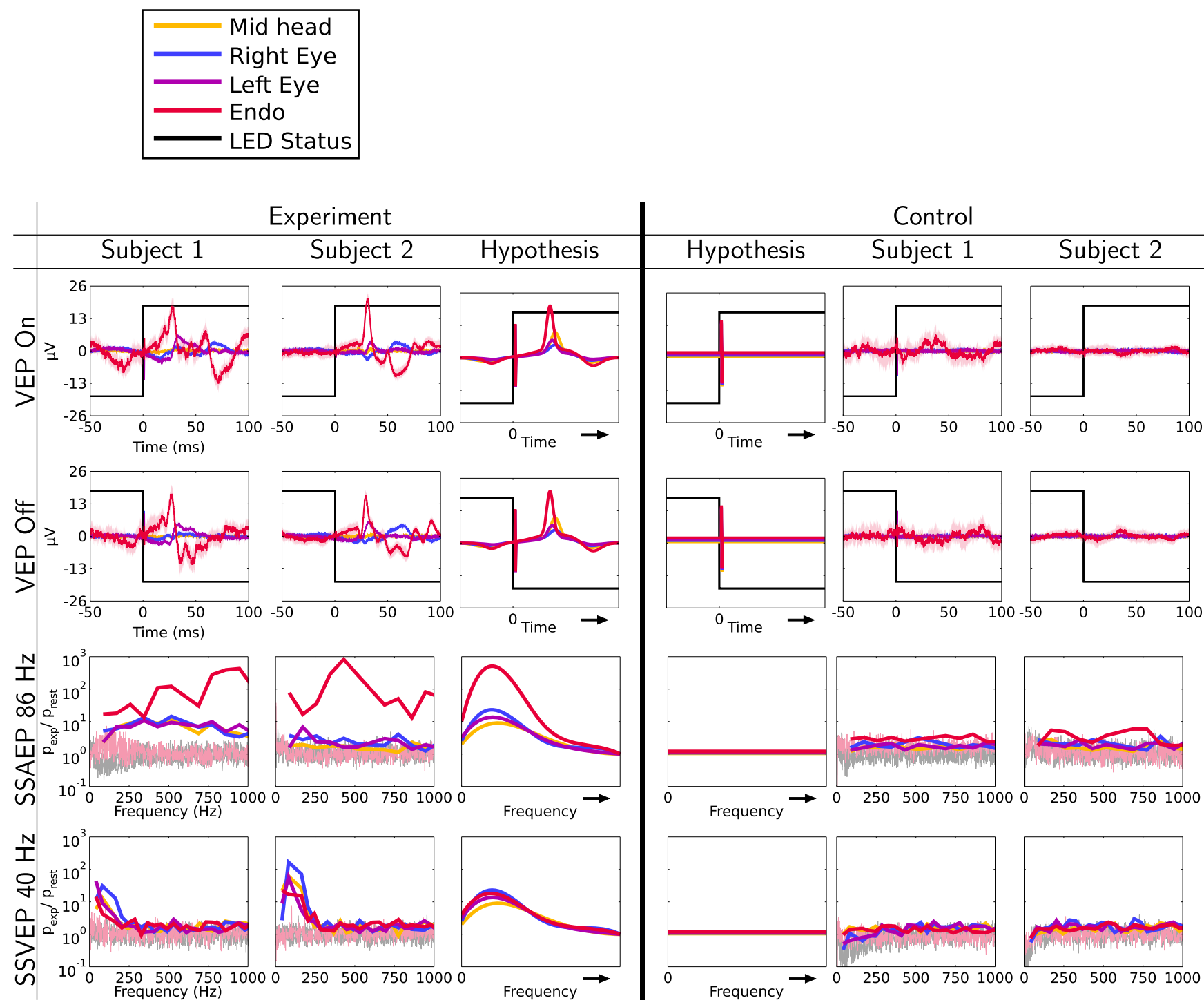

Figure 2. Performance of the electrodes at the optimal location for the endovascular location compared with the performance on the live control trials. The SSAEP and SSVEP experimental trials for Subject 1 are shown for the mid-basilar location. All other performances are shown at the basilar tip position. In each of the experiments, all experimental trials elicited a much larger response than the control experiments, which indicates that true non-artifactual responses were recorded. In addition to higher amplitude VEP signals, the endovascular electrode achieved better SNR compared to the scalp electrodes for the SSAEP experiments, and only scalp-comparable SNR for the SSVEP experiments. The hypothesis waveforms are qualitative, on the basis of proximity to areas previously attributed to the specific evoked potentials as described in results.

stimulus frequency with a 50\% duty cycle. A digital synchronization pulse was issued to the data acquisition system at the beginning and end of the stimulation period, but not during the stimulation period to avoid cross-talk-induced artifact between digital input and analog channels. Data was recorded in temporally contiguous stimulus and rest periods to compare stimulus-related neural response against temporally-contiguous resting-state neural signals. All stimulus periods lasted between $22.5 \mathrm{~s}$ and $57.0 \mathrm{~s}$, and all rest periods lasted between $28.8 \mathrm{~s}$ and $60.7 \mathrm{~s}$. In figure 2, data from the SSVEP experiment constitutes $22.5 \mathrm{~s}$ of stimulus and $30.6 \mathrm{~s}$ of rest in Subject 1, and 27.5 s of stimulus and $30.3 \mathrm{~s}$ of rest in Subject 2. Similarly, the SSVEP control constitutes $27.5 \mathrm{~s}$ of stimulus and $31.2 \mathrm{~s}$ of rest in Subject 1, and $27.5 \mathrm{~s}$ of stimulus and $30.1 \mathrm{~s}$ of rest in Subject 2. In figure 3, data from the SSVEP experiment consists of 22.5 to $57.0 \mathrm{~s}$ of stimulus and 30.4 to $60.7 \mathrm{~s}$ of rest, and data from the SSVEP control consists of 27.5 to $57.0 \mathrm{~s}$ of stimulus and 31.2 to $60.2 \mathrm{~s}$ of rest for Subject 1 .

SSAEP. The SSAEP was recorded by delivering $86 \mathrm{~Hz}$ auditory click trains through a standard earbud seated in the right external auditory canal. Clicks were produced as a train of monophasic $2 \mathrm{~ms}$ square wave pulses, generated every $11.625 \mathrm{~ms}$. Output from the computer to the earbud was amplified using the Dayton Audio DTA-120 Class T Digital Mini Amplifier. While audio levels were unchanged over the course of the experiment, absolute in-ear audio levels were not quantified. As with the SSVEP, a digital synchronization pulse recorded the start and end of the stimulation period. 


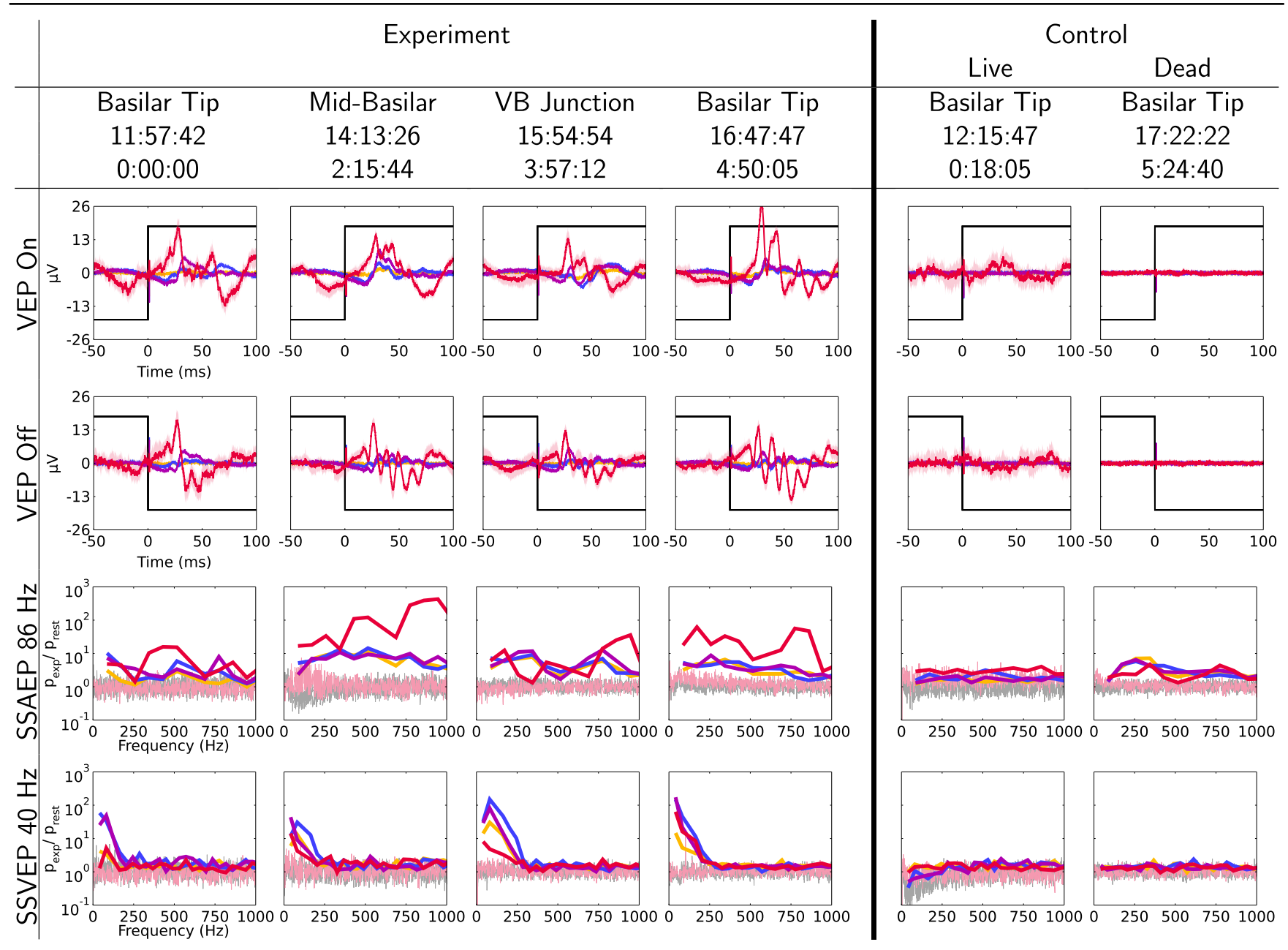

Figure 3. Demonstration of position dependence for Subject 1.The performance of the endovascular electrode varies as its location is changed. The first row of $24 \mathrm{~h}$ format times indicates the approximate time of recording for the corresponding column in local time. Induction of anesthesia occurred at 07:35 and euthanasia occurred at 17:01. The second row of times is provided relative to the first basilar tip recording. Where nearly $4 \mathrm{~h}$ passed between the basilar tip and VB junction recordings, the second basilar tip recording at $4 \mathrm{~h}$ and $50 \mathrm{~min}$ confirms that the brain continues to provide similar or higher quality evoked potentials relative to the first basilar tip recording. Live and dead animal control trials confirm that patterns observed in the experimental panels cannot be explained by non-brain biological signals (live control) or non-biological artifact (dead control).

Data was again recorded in temporally contiguous stimulus and rest periods to compare stimulus-related neural response against temporally-contiguous resting-state neural signals. All stimulus periods lasted between $29.0 \mathrm{~s}$ and $59.2 \mathrm{~s}$, and all rest periods lasted between $14.2 \mathrm{~s}$ and $59.0 \mathrm{~s}$. In figure 2, data from the SSAEP experiment constitutes $29.7 \mathrm{~s}$ of stimulus and $28.4 \mathrm{~s}$ of rest in Subject 1, and $53.6 \mathrm{~s}$ of stimulus and $23.5 \mathrm{~s}$ of rest in Subject 2. Similarly, the SSAEP control constitutes $29.2 \mathrm{~s}$ of stimulus and $28.8 \mathrm{~s}$ of rest in Subject 1, and $51.1 \mathrm{~s}$ of stimulus and $14.2 \mathrm{~s}$ of rest in Subject 2. In figure 3, data from the SSAEP experiment consists of 29.0 to $59.2 \mathrm{~s}$ of stimulus and 28.4 to $59.0 \mathrm{~s}$ of rest, and data from the SSAEP control consists of 29.2 to $59.0 \mathrm{~s}$ of stimulus and 28.8 to $59.0 \mathrm{~s}$ of rest for Subject 1.

Power spectrum analysis for SSVEP and SSAEP. SSVEP and SSAEP power spectral analyses were similar. For both visual and auditory experimental conditions, data was collected in stimulus periods and in temporally contiguous rest periods. For each period, Welsh's method was used to compute the power spectral density with a window length of $2 \mathrm{~s}$ and an overlap of $25 \%$. All data within a $3 \mathrm{~Hz}$ locus of $60 \mathrm{~Hz}$ and its harmonics were attributed to electrical noise and excluded from analysis. We then calculated a SNR at each frequency, given by the power in the neural signal measured during stimulus period divided by the neural signal power measured during the rest period.

The SNR was plotted in different colors for stimulus frequencies and non-stimulus frequencies. Stimulus frequencies were defined as all frequencies within a $3 \mathrm{~Hz}$ locus of the stimulus frequency and its harmonics. Non-stimulus frequencies were all remaining frequencies, excluding $60 \mathrm{~Hz}$ and harmonics. In the frequency domain plots (figures 2 and 3), stimulus frequencies are plotted in dark colors, and nonstimulus frequencies are plotted in lighter colors. All scalp electrodes from non-stimulus frequencies are plotted in gray for ease of visualization. The band of color demarcated by the non-stimulus frequencies on these plots serves as confidence 
(A)

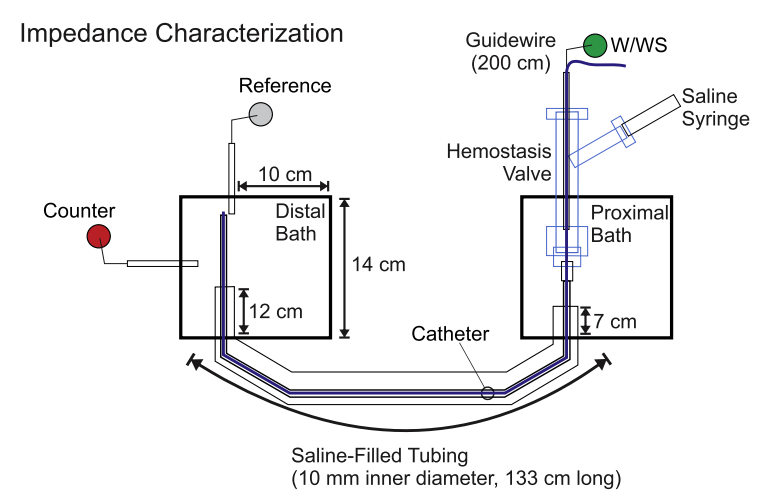

(C)

\section{System Characterization}

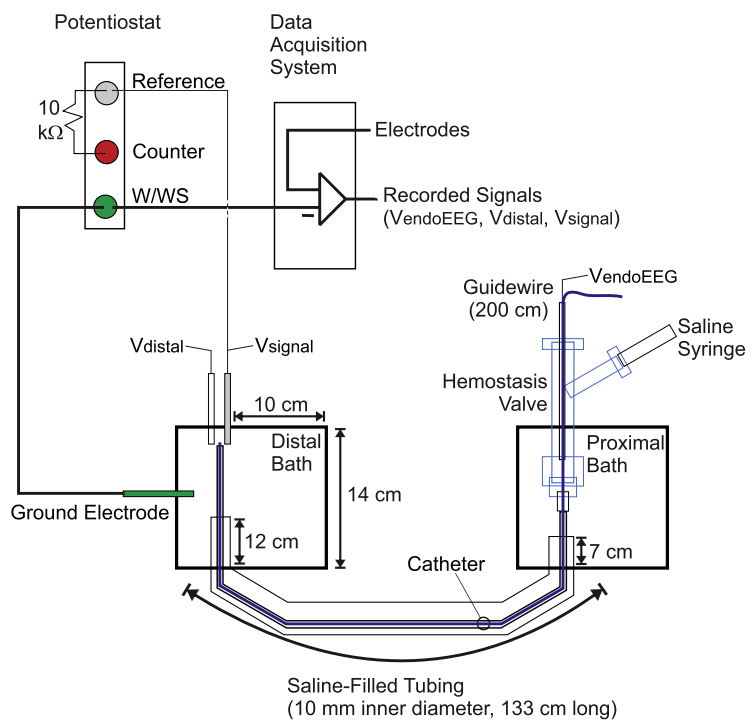

(B)
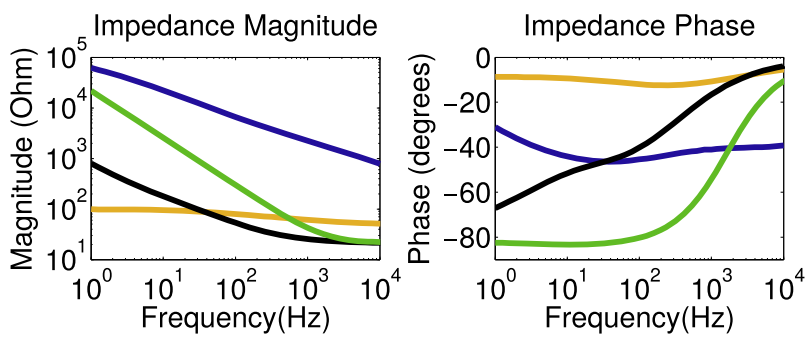

(D)
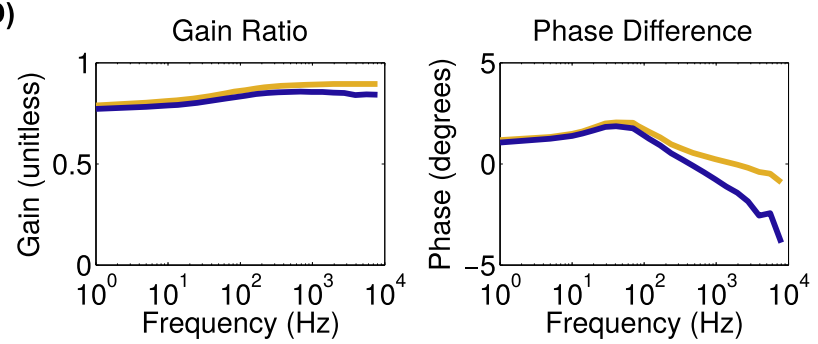

Figure 4. Impedance characterization. (A) Standard three-electrode impedance mea-surement of the guidewire electrode in a simulated endovascular setting. (B) Magnitude and phase of the impedance for the endovascular guidewire electrode compares unfavorably versus standard bioelectrode materials. (C) The system characterization apparatus applies a sinusoidal signal source $a \sin (2 \pi f+b)+c$ with amplitude $a$, phase $b$ and offset $c$ at the distal bath to compare the voltage recorded near the signal source with the voltage at the proximal guidewire. The system being characterized includes the guidewire electrode in series with the amplifier of the data acquisition system. (D) System characterization is expressed as the gain ratio $\left(a_{\text {endo }} / a_{\text {signal }}\right)$ and phase difference $\left(b_{\text {endo }}-b_{\text {signal }}\right)$ for the guidewire electrode, and similarly for $a_{\text {distal }}$ and $b_{\text {distal }}$ for an $\mathrm{Ag} / \mathrm{AgCl}$ electrode placed in the distal bath for comparison.

intervals in the assessment of the stimulus frequency curves. Specifically, if stimulus frequency curves exceed nonstimulus frequency confidence intervals, the signal-to-noise is considered to be statistically significant with at least $p<0.05$ on the basis of permutation testing (Efron and Tibshirani 1993).

\section{Cardiac artifact removal}

The endovascular electrode demonstrated cardiac artifact, nearly equal in amplitude to the precordial cardiac potential, where this artifact was nearly absent in scalp leads. This is illustrated for the mid-basilar position in subject 1 (figure 6). Average cardiac contamination potentials were calculated for each electrode, aligned to the R peak of the QRS complex measured on a precordial lead with reference to the nose electrode (figure 6(A)). These R-peak-averaged waveforms were calculated using 1076 QRS events and $180 \mathrm{~ms}$ analysis windows. Below, we describe our procedure for removing cardiac contamination from endovascular and scalp recordings.

In order to remove cardiac contamination from the raw traces (figure 6(B)), the precordial trace was first high-passed with a Butterworth filter (stopband at $2 \mathrm{~Hz}$, passband at $10 \mathrm{~Hz}$, stopband attenuation $90 \mathrm{~dB}$, passband ripple of $1 \mathrm{~dB}$ ) to remove slow drifts in the cardiac signal. R-peaks were marked as the time of maximum signal in each non-overlapping $180 \mathrm{~ms}$ interval. This interval length was chosen with consideration to the heart rate which typically varied between 265 and 285 beats per minute (one pulse every 210 to 


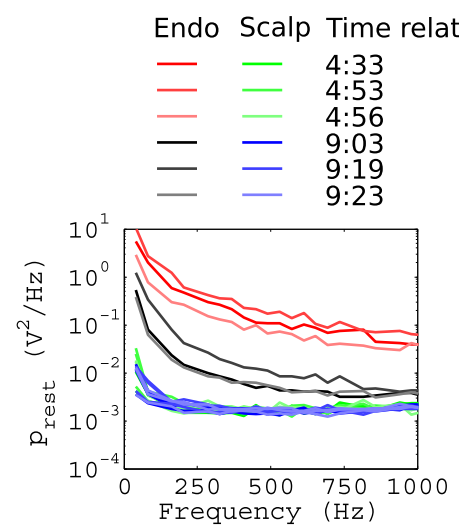

(A)

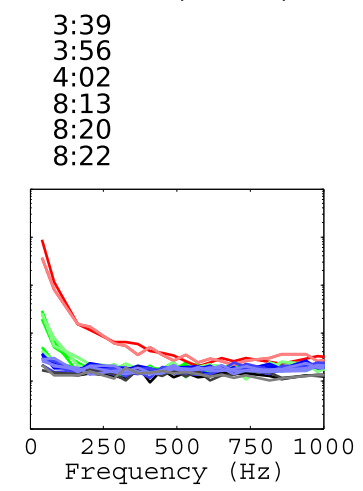

(B)

Figure 5. Non-stationarity in baseline response. The baseline power spectral density is shown for (A) Subject 1 and (B) Subject 2 at various points in time relative to induction of anesthesia for both scalp and endovascular electrodes at the basilar tip. The overall power diminishes in the endovascular electrode over hours, but remains stable on the scalp electrodes. The reason for this non-stationarity could be multifactorial, including causes related to the underlying biological substrate or the chemistry of the electrode-electrolyte interface.

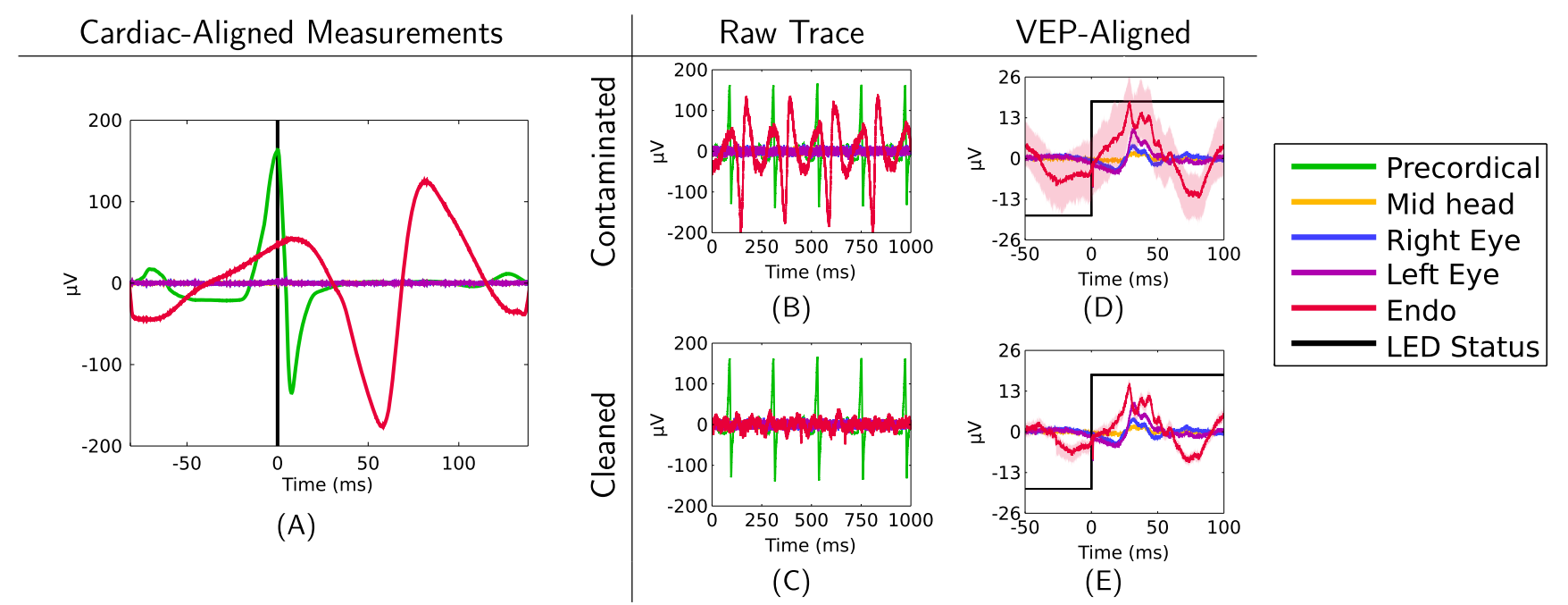

Figure 6. Cardiac contamination and cleaning. (A) The cardiac-aligned measurements show the mean (solid colors) and $95 \%$ bootstrap confidence intervals on the mean (shaded colors; barely perceptible beyond the mean) for 1076 heart beats. This endovascular electrode from Subject 2 is located at the mid-basilar location, which demonstrated the largest con-tamination from cardiac activity. The P-wave, QRS complex, and T-wave are all visible in the precordial (cardiac) electrode waveform (green). The raw endovascular electrode waveform also show highly repeatable cardiac artifacts (B), which are removed in the cleaned version (C). The VEP-aligned data has the same general trend before and after cardiac artifacts are accounted for, but trial-to-trial variability, represented in bootstrapped confidence intervals on the mean (light shading), is much larger before accounting for cardiac artifacts (D) than after (E).

$225 \mathrm{~ms}$ ). Next, $180 \mathrm{~ms}$ analysis intervals were selected from the endovascular and scalp electrodes based on R-peak times on the pre-cordial lead. These analysis intervals extended from $67 \mathrm{~ms}$ prior to each $\mathrm{R}$ peak (37\% of the analysis interval, systole) to $113 \mathrm{~ms}$ following each $\mathrm{R}$ peak $(63 \%$ of the analysis interval, diastole). Time zero in these analysis intervals represents the time of the $\mathrm{R}$ peak. Because the $\mathrm{R}-\mathrm{R}$ interval varied slightly in duration, shorter intervals were zero padded before averaging so that all intervals would be the same length. Finally, analysis intervals were averaged to produce a mean R-aligned cardiac contamination waveform for each electrode (figure 6(A)), which was then aligned with the raw data at $\mathrm{R}$ peaks and subtracted from the raw amplitudes to produce the cardiac-removed waveforms (figure 6(C)). The raw VEP-aligned endovascular electrode waveform demonstrates large trial-to-trial variability (figure 6(D)) which is dramatically reduced following removal of cardiac artifact (figure 6(E)).

\section{Electrode characterization}

In order to better delineate whether advantages of endovascular recording could be attributed to the endovascular positioning rather than differences in electrode properties, we 
characterized the guidewire electrode versus $\mathrm{Ag} / \mathrm{AgCl}$, platinum, and platinum black electrodes in physiological $(0.9 \%$, isotonic) saline solution.

To achieve guidewire electrical characterization under realistic conditions, we constructed a two-bath system connected by tubing (figures $4(\mathrm{~A})$ ). Using this system, the $\mathrm{X}$-Pedion-10 guide wire was passed in the usual fashion through the Echelon-10 microcatheter. A hemostasis valve was attached to the microcatheter hub, and the sidearm was loaded with a saline syringe. This configuration ensured that the full extent of the microcatheter exterior, microcatheter lumen, and guidewire remained fully submerged in saline without intervening air bubbles.

Prior work on metal microelectrodes highlighted the importance of performing full complex impedance characterization across a wide range of frequencies to understand signal distortion, rather than more commonly reported singlefrequency resistance values (Nelson et al 2008). Following this example, we performed both full impedance characterization and system characterization, both described below. Both measurements were made using the Reference 600 (Gamry Instruments, Inc.) for impedance spectroscopy, potentiostatic control, and electroplating.

Electrode preparation. Various benchmark electrodes were prepared for comparison with the guidewire electrode, including $\mathrm{Ag} / \mathrm{AgCl}$, platinum, and platinum black electrodes. Premade $\mathrm{Ag} / \mathrm{AgCl}$ electrodes (BASi, RE-5B $\mathrm{Ag} / \mathrm{AgCl}$ Reference Electrode with Flexible Connector) were removed from their glass housing with a glass cutter. The exposed length of $\mathrm{Ag} / \mathrm{AgCl}$ electrode was not controlled precisely. The submerged length was approximately $35 \mathrm{~mm}$.

Bare $0.5 \mathrm{~mm}$ diameter platinum wire served as the platinum electrode. Platinum black electrodes were prepared by electroplating this platinum wire in the following manner. The platinum wire was first cleaned with isopropyl alcohol, acetone, and sulfuric acid (1 $\mathrm{M} \mathrm{H}_{2} \mathrm{SO}_{4}$, Sigma-Aldrich), with intervening rinses using deionized water. Subsequently, the platinum wire was submerged in $10 \mathrm{mmol}$ chloroplatinic acid using a two-electrode configuration with a large-surface-area graphite return electrode. The voltage profile was initiated at $0 \mathrm{mV}$ for $5 \mathrm{~s}$, followed by $-260 \mathrm{mV}$ at $600 \mathrm{~s}$, using the Gamry chronoamperometry setting. Blackening of the exposed platinum, seen by eye, confirmed progression of the plating process. Both platinum black and bare-platinum wire were exposed to a precise length of $26.24 \mathrm{~mm}$ using multiple coats of clear nail polish to insulate the proximal extent.

\section{Impedance characterization. Complex impedance} measurements were performed with a standard threeelectrode configuration (figure 4(A-B)) using the Gamry Reference 600 instrument as an electrical impedance spectrometer. For $\mathrm{Ag} / \mathrm{AgCl}$, platinum and platinum black electrode measurements, all three electrodes (counter, reference, $\mathrm{W} / \mathrm{WS}$ ) were composed of the same material and placed in the distal bath. For guidewire measurements, $\mathrm{Ag} /$ $\mathrm{AgCl}$ reference and counter electrodes were used.

System characterization. We additionally examined the effect of electrode impedance on the overall ability for the data acquisition system to measure neural signals at different frequencies. This characterization was important to interpreting whether differences in performance in vivo resulted from intracranial electrode position or differences in guidewire versus scalp electrode material. To first approximation, the electrode in series with the data acquisition system is a voltage divider, so electrode materials that present with higher impedance magnitude would be expected to attenuate low-frequency neural signals to a greater extent. The extent of this difference depends on characteristics of the complete system, including the electrode and data acquisition system.

The system characterization setup (figure 4(C)) directly measured the extent of these differences in frequency attenuation between electrodes in the setting of our specific data acquisition system. To simulate a neural signal, a sine wave was applied to the distal bath between $\mathrm{V}_{\text {signal }}$ and the Ground Electrode using the Gamry Reference 600 instrument as a potentiostatic signal generator. Subsequently, the amplitude ratio and phase difference between $V_{\text {distal }}$ and $\mathrm{V}_{\text {endoEEG }}$ were measured (figure 4(D)). First, the $\mathrm{V}_{\text {distal }}$ and $\mathrm{V}_{\text {endoEEG }}$ signals are fit to sine curves. To accomplish this, the amplitude $a$, offset $b$, and phase $c$ are selected to minimize the squared error between the measured signal and the function $a \sin (2 \pi f+c)+b$. The estimated phases and amplitudes are then used to compute the amplitude ratio and the phase difference between $\mathrm{V}_{\text {distal }}$ and $\mathrm{V}_{\text {endoEEG }}$. For the endovascular electrode (endoEEG), the gain ratio is $\frac{a_{\text {endoEEG }}}{a_{\text {signal }}}$, and the phase difference is $c_{\text {endoEEG }}-c_{\text {signal }}$. For the $\mathrm{Ag} / \mathrm{AgCl}$ curve, the gain ratio is $\frac{a_{\text {distal }}}{a_{\text {signal }}}$, and the phase difference is $c_{\text {distal }}-c_{\text {signal }}$.

\section{Results}

In each of two subjects, multiple imaging modalities guided endovascular access and determined precise intracranial locations of the recording guidewire along the basilar artery. Representative images from the first subject (figure 1) illustrate the relevant anatomy. Digital subtraction angiography performed from a microcatheter injection of iodinated contrast at the vertebrobasilar (VB) junction (figure 1(A)) demonstrates the course of the basilar artery, terminating at the circle of Willis. This basilar artery gives off perforators that supply the brainstem, and two homologous superior cerebellar arteries that perfuse the cerebellum. For each recording, the guidewire was positioned just beyond the tip of the microcatheter, as confirmed by spot radiography (figure 1(B)). Precise location of the guidewire was confirmed with rotational computed tomography $(\mathrm{CT})$ on the procedure table, defining recording positions relative to the bony anatomy (figure 1(C)). 
Post-mortem structural MRI related this bony anatomy to the detailed brain parenchymal anatomy (figure 1(D)), including areas of the rabbit brain that are postulated to be involved in the production of auditory and VEPs. This minimal literature in rabbit suggests possible roles for the inferior colliculus in SSAEP (Hori et al 1993), occipital cortex in SSVEP (Hori et al 1992, Herrmann 2001), and both optic tracts/radiations and visual cortex in VEP (Arezzo et al 1988). Using the CT and MRI images to identify standard rabbit brain anatomy (Shek et al 1986), we see that recording positions along the basilar artery are slightly closer to the inferior colliculus and optic chiasm than visual cortex. Three-dimensional reconstruction of CT data illustrates the complete triaxial endovascular intracranial iEEG recording approach, with the guide catheter positioned at the osteum of the right vertebral artery, the microcatheter passing intracranially, and the guidewire electrode emerging at the tip of the microcatheter (figure $1(\mathrm{E})$ ).

Simultaneous guidewire electrode and scalp electrode recordings were performed at the basilar tip in two subjects, including VEP, SSVEP, and SSAEP experiments, followed immediately by live animal controls. Following cardiac artifact removal (see methods), multiple trials were aggregated to compute an average response and confidence intervals reflecting inter-trial response variability (figure 2 ). The two center columns represent hypotheses about the relative amplitude of evoked potentials confirmed in the flanking data columns for each of the two subjects. These hypotheses are qualitative and limited by minimal prior literature, based on proximity to corresponding brain centers believed to contribute to these evoked potentials in rabbit. Specifically, rabbit VEP is believed to include components from the retina, optic tracts and visual cortex (Arezzo et al 1988). Rabbit SSAEP signal generation is believed to predominate in the inferior colliculus (Hori et al 1993). Rabbit SSVEP signal generation is believed to result predominantly from visual cortex (Hori et al 1992). We identified the positions of these structures relative to the guidewire electrode on the basis of multimodal imaging (figure 1). Panel (D) shows that inferior colliculus and optic chiasm are closer to the basilar tip electrode than visual cortex. This ordering of distances is loosely preserved in human anatomy. Accordingly, we expected an ideal endovascular recording to dominate the scalp recordings in the VEP and SSAEP conditions, but not necessarily in the SSVEP condition where distance and intervening skull might exert countervailing effects on the ability for endovascular signal to dominate scalp recordings.

The VEP experimental data confirm previous trends from endovascular and subdural grid recordings that endovascular VEP amplitude is larger than scalp VEP. However, larger magnitude is not equivalent to improved signal quality. For example, multiplying the scalp EEG signal by a scaling factor would increase amplitude, but it would not affect SNR. Additionally, variability in signal amplitude at individual points of the VEP is not equivalent to SNR. This is because the signal amplitude variability cannot be easily attributed to noise versus greater variability in the neural response without learning the unknown probability distribution of the full VEP waveform. For example, the shapes of the endovascular and different scalp VEP responses are different, and this would need to enter into an analysis of SNR for VEP. These reasons may be in part why SNR comparisons have not been attempted on evoked potentials in the preceding four decades of literature in this area. Our steady-state evoked potential data for calculating SNR circumvents this problem by driving neural signals at known frequencies, avoiding the need to learn a high-dimensional neural response with limited data.

Our analysis of steady-state harmonics shows that SNR dominates for endovascular EEG over scalp EEG. The frequency domain analysis with SSAEP shows that the endovascular electrode preserves SNR (and not simply signal power) for a wider range of frequencies. This advantage in signal quality dissipates for SSVEP. This is possibly because the signal origin for SSVEP in visual cortex (Hori et al 1992) is more distant to the basilar tip than neural circuits participating in the VEP (including optic chiasm) (Arezzo et al 1988) and SSAEP (including the inferior colliculus) (Hori et al 1993), but a detailed deconstruction on the neural substrates for evoked potentials in rabbits is not presently available. Moreover, this distance-focused logic is not entirely complete because E-field magnitudes also depend on the projection of time-varying electric field vectors onto the displacement vector between measurement and recording electrodes (Geselowitz 1967), as routinely noted in electrocardiography (Mann et al 2014). Relative performance between endovascular and scalp SNR values in SSAEP and SSVEP are summarized as a ratio of these two quantities (table 1).

To directly test whether endovascular SNR demonstrated spatial dependence, we next examined three endovascular electrode positions along the basilar artery in subject 2 while recording the same evoked potentials (figure 3). Electrode position was retracted to sequentially interrogate the basilar tip, mid-basilar artery, and vertebrobasilar (VB) junction. For the final recording position, the guidewire electrode was restored to the approximate location of the basilar tip to confirm that diminishing endovascular recording amplitudes were not trivially a result of a time-dependent process unrelated to electrode position, like cerebral ischemia or electrochemical properties of the guidewire-electrolyte interface. Peri-procedural CT confirmed that the VB junction in subject 2 was located just at the entry to the foramen magnum, where the posterior cerebral vasculature enters the skull. As such, the VB junction recording location served as an interesting condition for assessing approximately-extracranial endovascular signal quality.

Plots at these positions show that endovascular positioning modulates amplitude of the evoked potential. For VEP, endovascular signal amplitude peaked at the basilar tip and progressively diminished for more retracted electrode positions. For SSAEP, the mid-basilar recording position produced markedly higher endovascular SNR than other positions. For SSVEP, endovascular SNR was less evidently modulated by electrode position. The return to basilar tip properly restored SNR from the diminished values at the VB junction, demonstrating that poor SNR at the VB junction was 
Table 1. Representative SNR ratios based on SSAEP and SSVEP responses. The SNR ratio is reported in these tables, defined by the endovascular electrode SNR divided by the best-performing scalp electrode SNR. The maximum, median, and minimum ratio for harmonics of the stimulus frequency from $0 \mathrm{~Hz}$ to $1000 \mathrm{~Hz}$ are computed. This range of frequencies corresponds to the interval shown in figures 2 and 3 . (A) Comparison of Subject 1 and Subject 2 at the best recording position, which was either mid-basilar or basilar tip. This corresponds to the data in figure 2. (B) Comparison of performance at various locations for Subject 1. This corresponds to the data in figure 3.

\begin{tabular}{llrr}
\hline & & Subject 1 & Subject 2 \\
\hline \multirow{3}{*}{ SSAEP } & Max & 76.1690 & 271.8237 \\
& Median & 2.5797 & 1.2750 \\
& Min & 0.7825 & 0.4387 \\
\hline \multirow{3}{*}{ SSVEP } & Max & 1.5005 & 1.8038 \\
& Median & 0.9234 & 0.8879 \\
& Min & 0.1386 & 0.1017 \\
\hline
\end{tabular}

(A)

\begin{tabular}{llcccr}
\hline & $\begin{array}{c}\text { Basilar tip } \\
11: 57: 42 \\
0: 00: 00\end{array}$ & $\begin{array}{c}\text { Mid-basilar } \\
14: 13: 26\end{array}$ & $\begin{array}{c}\text { VB junction } \\
15: 54: 54 \\
3: 57: 15: 44\end{array}$ & $\begin{array}{c}\text { Basilar tip } \\
16: 47: 47 \\
4: 50: 05\end{array}$ \\
\hline \multirow{2}{*}{ SSAEP } & Max & 8.1560 & 76.1690 & 1.5093 & 17.9343 \\
& Median & 1.1322 & 2.5797 & 0.9315 & 1.4521 \\
& Min & 0.3936 & 0.7825 & 0.0322 & 0.6284 \\
\hline \multirow{2}{*}{ SSVEP } & Max & 1.5386 & 1.5005 & 12.6037 & 1.4772 \\
& Median & 0.7677 & 0.9234 & 1.1315 & 0.8991 \\
& Min & 0.0218 & 0.1386 & 0.1127 & 0.3526 \\
\hline
\end{tabular}

(B)

unlikely to be the result of changing neural substrate or drifting electrode properties. The live and dead controls confirmed that signals derived from endovascular and other electrodes were not the result of non-neural biological signals or electrical crosstalk.

We next investigated whether the improved SNR and wider frequency range manifest by endovascular intracranial recording positions over scalp recording could be plausibly attributed to differences in material composition rather than differences in location. Because guidewire electrical properties are entirely unknown, we performed in vitro electrochemical measurements (figure 4), previously described in the neuroscience literature with standard metal electrodes (Nelson et al 2008). We first measured and compared impendence spectra of our guidewire/catheter system versus $\mathrm{Ag} / \mathrm{AgCl}$, platinum, and platinum black electrodes. Using a saline bath customized to accommodate the catheter and guide wire electrode (figure 4(A)), we performed a standard three-electrode impedance measurement (figure 4(B)). The measurements show that the guide wire electrode results in nearly three orders of magnitude larger impedance over all frequency ranges than $\mathrm{Ag} / \mathrm{AgCl}$, which is the composition of our scalp electrodes. Moreover, the guidewire electrode impedance is between one and three orders of magnitude larger than bare platinum and demonstrates impedance phase non-linearity. This impedance data shows that these endovascular recordings outperformed scalp recordings despite unfavorable impedance characteristics of the guidewire electrode. In a subsequent in vitro setup (figure 4(C)), we characterized the guidewire electrode in series with the data acquisition amplifier, to determine whether the intrinsic impedance characteristics of the electrode had tangible bearing on the overall ability for the electrode to capture neural data at a range of frequencies. In comparison with $\mathrm{Ag} / \mathrm{AgCl}$ we found only minimal magnitude and phase distortion with higher frequencies. As expected, the high input impedance of our recording system partially compensated differences between impedances of the various electrodes.

\section{Discussion}

In comparison with non-invasive scalp EEG, intracranial recordings provide wider frequency content and improved spatial resolution. For some patients with tumor or epilepsy requiring surgery near eloquent cortex or presenting with ambiguous scalp data, this improvement in signal quality warrants open-skull surgery to implant subdural electrode arrays for up to two weeks. For the vast majority of patients suffering from functional brain diseases, the considerable discomfort and risk of major complications associated with subdural grid electrodes places this level of signal quality out of reach.

In the meantime, endovascular surgical techniques have dramatically advanced our ability to safely access the brain without craniotomy. Intermittent studies over the past four decades demonstrated that endovascular intracranial EEG recordings could provide larger-magnitude neural signals than 
scalp recordings, but the actual quality of these signals has remained unknown. In the present work, we exploited both time- and frequency-domain analysis of evoked potentials to demonstrate critically that endovascular EEG provides higher quality signals than scalp recordings, in a spatially-dependent fashion.

This work also demonstrated the feasibility of rabbit as a tractable small-animal model. We combined trans-femoral endovascular access, cross-sectional imaging, and multi-sensory evoked potentials, all within the same test subjects. The rabbit arterial anatomy closely resembles human, in contrast to sheep or pig (Mehra et al 2012). The possibility of liverabbit fMRI has been demonstrated elsewhere, which will be an important adjunct to localize areas of stimulus-dependant neuromodulation (Wyrwicz et al 2000). Although rabbit arteries are more prone to vasospasm than human subjects, we found that vasospasm in our subjects resolved over a period of several minutes without pharmacological intervention. Moreover, we were able to use a larger and more durable microcatheter than previously described (Culp et al 2007, Jahan et al 2008). For some studies, the lissencephalic cortical anatomy or availability of specific genetic mutations will be important limitations of the rabbit model. Additionally, venous access to the brain is more easily achieved in larger animal models.

Various complementary techniques could benefit from acute or chronic endovascular recording. Semi-chronic highdensity electrode arrays placed within the dural venous sinuses could provide a realistic minimally-invasive alternative for patients that would otherwise undergo craniotomy for pre-surgical epilepsy monitoring. Although venous infarcts are possible, venous implantation would minimize the risk of arterial stroke while providing access to both cortical and deep brain structures. Unlike craniotomy-based iEEG, acute intra-operative monitoring with endovascular EEG could be performed entirely without anesthesia. With the growing diversity of neurostimulation techniques such as focused ultrasound, intraparenchymal electrodes, and optogenetics, there is an imperative to enable chronic, highquality, real-time feedback to these devices without requiring craniotomy. Ambiguities introduced by pre-surgical fMRI could be investigated and resolved with a targeted endovascular brain mapping study. Endovascular EEG during intracarotid sodium amobarbital administration and cognitive testing could significantly enrich the Wada test where endovascular catheters are already present. The capability for measuring local brain activity could provide rapid and precise feedback during endovascular embolization of tumors, aneurysms, and vascular malformations. Analogous to focal cortical stimulation before surgical resection, endovascular EEG during these embolization procedures could provide a high-SNR, frequency-rich, spatially sensitive adjunct to whole-brain scalp EEG in characterizing evoked potentials during intra-arterial injections of ultrashort-acting barbiturates in anesthetized patients.

In the near term, the development of endovascular neural recording and stimulation represents an exciting interdisciplinary endeavor with strong potential for patient impact.
Development in this area will leverage experience with other traditional and emerging devices like pacemakers, flowdiverting stents, microcatheters, and flexible-substrate electrode arrays. Standard clinical issues should be anticipated, including patient selection, decision to anticoagulate, endovascular device delivery, cellular and immune response, acute versus chronic recording quality, outcomes assessment, and cost effectiveness. Engineering development will incorporate insights related to materials, implantable electronics design, signal processing, control theory, and machine learning. The path to this goal will undoubtedly engage a wide array of partnerships, connecting researchers in signal processing, hardware design, materials science, neuroscience, and medicine.

\section{Acknowledgments}

Funding was provided in part to BDH by the Caltech Summer Undergraduate Research Fellowship, and to LS by UCLA Radiology and Stanford Radiology. We would like to thank the staff of the UCLA Translational Research Imaging Center, as well as Sandra Duarte-Vogel, DVM for assistance during experiments. Thanks to Amit Balgude, MD for discussions on endovascular access. Thanks to Luis Armendariz and Louis Yang from the Neural Signal Processing Laboratory (NSPL) for early theoretical feasibility analysis with current dipole modeling. Thanks to Siamak Yousefi and Christopher Hallacy (NSPL) for early experimental planning. Thanks to Rengaswamy Srinivasan, Applied Physics Laboratory, for discussions on impedance characterization.

\section{References}

Arezzo J C, Brosnan C F, Schroeder C E, Litwak M S and Bornstein M B 1988 Electrophysiological analysis of factors involved in the primary demyelinating diseases: the rabbit eye model system Brain Res. 462 286-300

Arya R, Mangano F T, Horn P S, Holland K D, Rose D F and Glauser T A 2013 Adverse events related to extraoperative invasive EEG monitoring with subdural grid electrodes: a systematic review and meta-analysis Epilepsia 54 828-39

Asirvatham S J, Friedman P A, Bruce C J and Holmes D R Jr 2012 Detecting and treating nervous system disorders US Patent Specification US8812099 B2

Ben-Haim S, Asaad W F, Gale J T and Eskandar E N 2009 Risk factors for hemorrhage during microelectrode-guided deep brain stimulation and the introduction of an improved microelectrode design Neurosurgery 64 754-62

Boniface S and Antoun N 1997 Endovascular electroencephalography: the technique and its application during carotid amytal assessment J. Neurology Neurosurgery Psychiatry 62 193-5

Bower M R, Stead M, Van Gompel J J, Bower R S, Sulc V, Asirvatham S J and Worrell G A 2013 Intravenous recording of intracranial, broadband EEG $J$. Neurosci. Methods 214 21-6

Caldwell B, Flores R, Lowery J, Brown A T and Culp W C 2011 Variations in the circle of Willis in the New Zealand white rabbit J. Vascular Interventional Radiol.: JVIR 22 1188-92

Chan D, Zhu X L, Yeung J H, Mok V C, Wong E, Lau C, Wong R, Lau C and Poon W S 2009 Complications of deep brain stimulation: a collective review Asian J. Surg. 32 258-63 
Cloft H J, Joseph G J and Dion J E 1999 Risk of cerebral angiography in patients with subarachnoid hemorrhage, cerebral aneurysm, and arteriovenous malformation a metaanalysis Stroke 30 317-20

Culp B C, Brown A T, Erdem E, Lowery J and Culp W C 2007 Selective intracranial magnification angiography of the rabbit: basic techniques and anatomy $J$. Vascular Interventional Radiol. 18 187-92

Driller J, Hilal S, Michelsen W, Sollish B, Katz B and Konig W Jr 1968 Development and use of the POD catheter in the cerebral vascular system Med. Res. Eng. 8 11-6

Efron B and Tibshirani R 1993 An Introduction to the Bootstrap (New York: Chapman and Hall)

Enomoto T, Matsumura A, Matsumaru Y, Aoki K and Nose T 2002 Multimodality examination for detection of the epileptic foci with special reference to endovascular EEG recording International Congress Series (Amsterdam: Elsevier) pp 655-60

Fiorella D, Hirsch J A, Woo H H, Rasmussen P A, Hussain M S, Hui F K, Frei D, Meyers P M, Jabbour P and Gonzalez L F 2012 Should neurointerventional fellowship training be suspended indefinitely? J. Neurointerventional Surg. 4 315-8

García-Asensio S, Guelbenzu S, Barrena R and Valero P 1999 Technical aspects of intra-arterial electroencephalogram recording Interventional Neuroradiology: J. Peritherapeutic Neuroradiology Surgical Proced. Relat. Neurosci. 5 289-300

Geselowitz D B 1967 On bioelectric potentials in an inhomogeneous volume conductor Biophys. J. 7 1-11

Henz B D, Friedman P A, Bruce C J, Holmes D R, Bower M, Madhavan M, DeSimone C V, Wahnschaffe D, Berhow S and Danielsen A J 2014 Advances in radiofrequency ablation of the cerebral cortex in primates using the venous system: improvements for treating epilepsy with catheter ablation technology. Epilepsy Res. 108 1026-31

Herrmann C S 2001 Human EEG responses to $1-100 \mathrm{~Hz}$ flicker: resonance phenomena in visual cortex and their potential correlation to cognitive phenomena Exp. Brain Res. 137 346-53

Hori A, Yasuhara A and Yasuhara M 1992 Origins of steady-state visual evoked potentials to flash stimulation in rabbits derived by source derivation technique and coherence analysis J. Kansai Med. Univ. 44 S35-44

Hori A, Yasuhara A, Naito H and Yasuhara M 1993 Steady-state auditory evoked potentials (SSAEPs) in the rabbit. Contribution of the inferior colliculus Electroencephalogr. Clin. Neurophysiol. 88 229-36

Ishida S, Shimamoto H, Abe T, Motooka H, Sakurai S, Takayama K, Yamaguchi H and Maeda H 1998 Intracranial EEG recording from intravascular electrodes in patients with temporal lobe epilepsy Epilepsia 39 77-77

Jahan R, Stewart D, Vinters H V, Yong W, Vinuela F, Vandeberg P and Marder V J 2008 Middle cerebral artery occlusion in the rabbit using selective angiography application for assessment of thrombolysis Stroke 39 1613-5

Kern M, Aertsen A, Schulze-Bonhage A and Ball T 2013 Heart cycle-related effects on event-related potentials, spectral power changes, and connectivity patterns in the human ECoG Neuroimage 81 178-90

Kunieda T, Ikeda A, Mikuni N, Ohara S, Sadato A, Taki W, Hashimoto N and Shibasaki H 2000 Use of cavernous sinus EEG in the detection of seizure onset and spread in mesial temporal lobe epilepsy Epilepsia 41 1411-9

Litt B and Echauz J 2007 Method and device for the recording, localization and stimulation-based mapping of epileptic seizures and brain function utilizing the intracranial and extracranial cerebral vasculature and/or central and/or peripheral nervous system US Patent Specification US20080027346 A1

Llinas R R 2007 Brain-machine interface systems and methods US Patent Specification US8447392 B2
Llinas R R, Walton K D, Nakao M, Hunter I and Anquetil P A 2005 Neuro-vascular central nervous recording/stimulating system: using nanotechnology probes $J$. of Nanoparticle Research 7 $111-27$

Lozano A M, Gildenberg P L and Tasker R R 2009 Textbook of Stereotactic and Functional Neurosurgery (Berlin: Springer)

Mann D L, Zipes D P, Libby P and Bonow R O 2014 Braunwald's Heart Disease: A Textbook of Cardiovascular Medicine (Amsterdam : Elsevier)

Mehra M, Henninger N, Hirsch J A, Chueh J, Wakhloo A K and Gounis M J 2012 Preclinical acute ischemic stroke modeling J. Neurointerv. Surg. 4 307-13

Mikuni N, Ikeda A, Murao K, Terada K, Nakahara I, Taki W, Kikuchi H and Shibasaki H 1997 'Cavernous sinus EEG': a new method for the preoperative evaluation of temporal lobe epilepsy Epilepsia 38 472-82

Müllhaupt D, Augsburger H, Schwarz A, Fischer G, Kircher P, Hatt J M and Ohlerth S 2015 Magnetic resonance imaging anatomy of the rabbit brain at $3 \mathrm{~T}$ Acta Veterinaria Scand. 57 $1-9$

Muñoz-Moreno E, Arbat-Plana A, Batalle D, Soria G, Illa M, Prats-Galino A, Eixarch E and Gratacos E 2013 A magnetic resonance image based atlas of the rabbit brain for automatic parcellation PloS One 8 e 67418

Nakase H, Ohnishi H, Touho H, Karasawa J, Yamamoto S and Shimizu K 1995 An intra-arterial electrode for intracranial electro-encephalogram recordings Acta Neurochirurgica 136 $103-5$

Nelson M J, Pouget P, Nilsen E A, Patten C D and Schall J D 2008 Review of signal distortion through metal microelectrode recording circuits and filters $J$. Neurosci. Methods 169 141-57

Nowell M, Miserocchi A, McEvoy A W and Duncan J S 2014 Advances in epilepsy surgery J. Neurology Neurosurgery Psychiatry 85 1273-9

Penn R D, Hilal S K, Michelsen W J, Goldensohn E S and Driller J 1973 Intravascular intracranial EEG recording: technical note J. Neurosurgery 38 239-43

Richard P and Urban I 1972 A Stereotaxic Atlas of the New Zealand Rabbit's Brain (Springfield, IL: Chanes C Thomas)

Ruddy B P 2006 Conducting polymer wires for intravascular neural recording Doctoral Dissertation Massachusetts Institute of Technology Cambridge, MA

Schalk G and Leuthardt E C 2011 Brain-computer interfaces using electrocorticographic signals IEEE Rev. Biomed. Eng. 4 140-54

Schiltz N K, Koroukian S M, Lhatoo S D and Kaiboriboon K 2013 Temporal trends in pre-surgical evaluations and epilepsy surgery in the US from 1998 to 2009 Epilepsy Res. 103 270-8

Scremin O U, Sonnenschein R R and Rubinstein E H 1982 Cerebrovascular anatomy and blood flow measurements in the rabbit J. Cerebral Blood Flow Metabolism: Official J. Int. Soc. Cerebral Blood Flow Metabolism 2 55-66

Shah A K and Mittal S 2014 Invasive electroencephalography monitoring: indications and presurgical planning Ann. Indian Acad. Neurology 17 S89

Shek J W, Wen G Y and Wisniewski H M 1986 Atlas of The Rabbit Brain and Spinal Cord (Basel: Karger)

Sheth S A, Aronson J P, Shafi M M, Phillips H, Velez-Ruiz N, Walcott B P, Kwon C S, Mian M K, Dykstra A R and Cole A 2014 Utility of foramen ovale electrodes in mesial temporal epilepsy Epilepsia 55 713-24

Simon M V 2009 Intraoperative Neurophysiology: A Comprehensive Guide to Monitoring and Mapping (New York: Demos Medical Publishing)

Stoeter P, Dieterle L, Meyer A and Prey N 1995 Intracranial electroencephalographic and evoked-potential recording from intravascular guide wires Am. J. Neuroradiology 16 1214-7

Thomke F, Stoeter P and Stader D 1998 Endovascular electroencephalography during an intracarotid amobarbital test 
with simultaneous recordings from 16 electrodes J. Neurology Neurosurgery Psychiatry 64 565-565

Voytek B, Secundo L, Bidet-Caulet A, Scabini D, Stiver S I,

Gean A D, Manley G T and Knight R T 2010

Hemicraniectomy: a new model for human electrophysiology with high spatio-temporal resolution J. Cogn. Neurosci. 22 2491-502

Watanabe $\mathrm{H}$, Takahashi $\mathrm{H}$, Nakao M, Walton $\mathrm{K}$ and Llinás R R 2009 Intravascular neural interface with nanowire electrode Electron. Commun. Japan 92 29-37
Wellmer J, von der Groeben F, Klarmann U, Weber C, Elger C E, Urbach H, Clusmann H and von Lehe M 2012 Risks and benefits of invasive epilepsy surgery workup with implanted subdural and depth electrodes Epilepsia 53 1322-32

Wyrwicz A M, Chen N K, Li L, Weiss C and Disterhoft J F 2000 fMRI of visual system activation in the conscious rabbit Magn. Reson. Med. 44 474-8

Zeitlhofer J, Feldner G, Heimberger K, Mayr N and Samec P 1990 Transarterielles EEG bei superselektiver zerebraler Angiographie Klin Neurophysiol. 21 70-2 